\title{
On the Formation of the New Construction "ta bu xiang ma" of Polysemy Isomorphism and Its Usage in Discourse
}

\author{
Min Zhao \\ Shanghai International Studies University, Shanghai, China
}

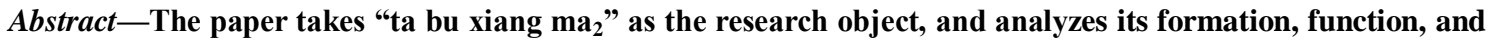
application in discourse. The study results indicate that "ta bu xiang $\mathrm{ma}_{2}$ " is a new and fillable construction that expresses the meaning of the domain of knowing and uttering. The structure has the functions of subjectivity, exclamation, anticipation and illocutionary. The formation mechanism is metaphor, and the motivation is the widespread use of network language and people's psychology of seeking novelty and differences. "Ta bu xiang ma" has strong spoken language style and is used in interactive and evaluative contexts. It can be used as both trigger and response sentences, and it has similarities and differences with its related formats.
\end{abstract}

Index Terms - polysemy isomorphism, rhetorical question construction, "ta bu xiang ma", spatial fusion

\section{INTRODUCTION}

In daily communication, rhetorical question is used frequently and it can be used as both trigger and response words. Rhetorical question has the function of interrogation, subjectivity and interactive subjectivity, anticipation, exclamation and illocutionary. Li Yufeng (2010), Zhu Jun (2013), etc. have conducted researches on rhetorical questions from the perspective of interactive communication. However, few people have studied the rhetorical expression "ta bu xiang ma (他不香吗)". Since 2019, with the spread of an old man's words “Is it not fragrant to buy pork ribs with the money from picking up bottles?" online, "ta bu xiang ma" has been widely used, and its uses are not limited to Internet terms, but gradually penetrate into people's daily communication. But when it is used in different contexts, the meaning of this expression is different, and the structure is also different. E.g:

(1) 百合花他不香吗? (自拟) ${ }^{1}$

Baihehua ta buxiang ma?

Lilies he not fragrant?

Don't lilies smell fragrant? (Self drafted)

（2）韭菜炒鸡蛋它不香吗？？？？？？（新浪微博 2020-06-20）

Jiucai chao jidan ta buxiang ma?

Scrambled Eggs with Leek it not delicious?

Aren’t Scrambled Eggs with Leek delicious? (Sina Weibo 2020-06-20)

（3）我的衣服已经够多了, 这些钱买吃的不香吗?（新浪微博 2020-06-06）

Wode yifu yijing gou duo le, zhexie qian mai chide buxiang ma?

My clothes already enough, this money buy something eat not fragrant?

I already have enough clothes. Isn't it good for buying something to eat with this money?

(Sina Weibo 2020-06-06)

In the above cases, "ta bu xiang ma" is a polysemy isomorphic form. In sentence (1) and sentence (2), "ta" and the subject "lily" and "Scrambled Eggs with Leek" form a homotopic relationship; "xiang" describes the smell and the flavour. "Ta bu xiang ma" is a negative form of rhetorical question. When questioning, it expresses affirmative meaning. In example (3), "ta bu xiang ma ${ }_{2}$ " is a buzzword on the Internet, and is used to make suggestions. The meaning of "ta bu xiang ma" in example (3) is "Is it good to buy food with money?" It can be seen that "ta bu xiang ma" has evolved from a rhetorical sentence to a standardized and solidified expression. So what are the formation mechanism and motivation? What discourse functions does this construction have? These are the questions to be discussed in this paper.

This paper takes "ta bu xiang $\mathrm{ma}_{2}$ " as the research object. Based on the analysis of its construction and discourse function, the paper discusses the evolution mechanism and motivation and the using context, and finally compares it with similar formats.

\footnotetext{
1 The corpus in this paper comes from Sina Weibo, Baidu search, and natural spoken dialogues. The online search period is from January 1,2019 to September 20, 2020. A few examples come from introspection. The source of all corpora is indicated, and punctuation and spaces strictly follow the original text.
} 


\section{CONSTRUCTION AND Discourse FunCTION}

The meaning expressed by the negative rhetorical question "ta bu xiang ma" can be directly deduced from its external expression: the basic grammatical meaning of the rhetorical question is "negation" (Lv Shuxiang, 1982), and the negative negation means affirmation, “xiang (香)" means "smells good, tastes good” (Dictionary editing room, Institute of Linguistics, Chinese Academy of Social Sciences, 2016, P. 1429). "Ta bu xiang ma ${ }_{1}$ " expresses affirmative meaning, refers to the fragrance one feels through smell and gustation directly. While the meaning of "ta bu xiang ma" cannot be understood literally, which has a rhetorical tone and means that when someone is obsessed with something that is not cost-effective, the other uses another options to persuade him to give up. According to Goldberg's construction grammar theory, as long as the overall meaning or function expressed by a certain language form cannot be fully deduced from its components, the expression can be regarded as a construction. "Ta bu xiang ma" has been constructed into a new and fillable construction with a rhetorical question as the construction and "ta" and "xiang" as the components. With the development and evolution of the construction, the meaning and discourse functions of "ta bu xiang $\mathrm{ma}_{2}$ " have also changed. According to Shen Jiaxuan (2003), language expression has three domains: acting, knowing and uttering domain. "Acting" refers to the behavior and action which is the description and expression of actual behavior; "knowing" refers to knowledge and cognition which is the speaker's inference and evaluation of reality; "uttering" refers to speech and utterance which is related to the speaker's communicative intentions. "Ta bu xiang ma," expresses the meaning of the acting domain, which is a description of the results of "smell" and "taste". The judgment of "fragrance" is mainly based on objective facts, and at the same time, subjectivity also plays some role. While "ta bu xiang $\mathrm{ma}_{2}$ " expresses the meaning of knowing and uttering domain, and it is the speaker's subjective judgment on "whether something is worth doing" which has the function of acting with words. "Ta bu xiang ma" is a way of speech act, with the subjectivity of the speaker. The evolution of the domain of acting from "ta bu xiang ma" to the domain of knowing and utting of "ta bu xiang $\mathrm{ma}_{2}$ " is consistent with the three-domain meaning proposed by Shen Jiaxuan (2003)_ _ acting domain is basic, knowing and uttering domain are derived from acting domain.

In addition to subjectivity, "ta bu xiang $\mathrm{ma}_{2}$ " also has the discourse functions of illocutionary, anticipation and exclamation. First of all, rhetorical questions express negative meaning. They are a kind of imperative sentences. While stating opinions and expressing attitudes, they also have the function of acting with words. "Ta bu xiang ma" is "words of action" and has the function of illocutionary. Second, the difference between affirmation and negation is that affirmation can have no presupposition, but negation always presupposes the corresponding positive content, and then negates or refutes it (Shen Jiaxuan, 1999). When the speaker uses "ta bu xiang ma", he first denies the listener's original opinions, and then puts forward his own suggestions and expects the listener to accept and adopt them. Therefore, "ta bu xiang $\mathrm{ma}_{2}$ " has the function of anticipation. When expressing in rhetorical questions, the speaker usually expresses the existing emotions and attitudes in the form of question, and the purpose is to enhance the expressive effect. Liu Yuehua (2001) believes that rhetorical questions use the rhetorical tone to affirm or deny a certain obvious truth or fact, in order to strengthen the tone of speech and express the emotion and attitude. Therefore, "ta bu xiang $\mathrm{ma}_{2}$ " also has the discourse function of expressing exclamation.

\section{FORMATION MECHANISM AND MotiVATION}

\section{A. Formation Mechanism}

The main formation mechanism of "ta bu xiang $\mathrm{ma}_{2}$ " is metaphor. Fauconnier (1997) proposed that metaphor is a cognitive process that relies on the cross-domain mapping between metaphorical objects and ontology, activates different conceptual information, and forms cross-domain connections under certain conditions, thereby generating new information (Wang Yin, 2007). We use Fauconnier and Turner's Theory of Conceptual Blending to explain the formation mechanism and formation process of "ta bu xiang $\mathrm{ma}_{2}$ ". Theory of Conceptual Blending believes that Conceptual Fusion includes one Generic Space, one Blended Space and two Input Spaces. The cognitive subject maps the two Input Spaces to the Fusion Space. Fusion Space integrates the received information to form new information. The Input Space 1 is equivalent to the source field, the Input Space 2 is equivalent to the target field, the Fusion Space is equivalent to the similar attributes and information in the source and target field, and the Fusion Space is equivalent to the final formed Image schema.

"Ta bu xiang $\mathrm{ma}_{2}$ " is a metaphorical cognitive process of new information formed by the component "xiang" from the concrete domain of taste and smell to the abstract domain, through reasoning and conceptual blending. "Xiang" in Input Space 1 means "smells good, tastes good", which can be directly perceived through sense; the fragrance is pleasant and enjoyable, from which can extract the conceptual attributes of "popular and valued" to form a generic space, and then this generic space attribute is projected to the Input Space 2- things with the attributes of "popular and valued" are worth doing and cost-effective. The related concepts in Input Space 1 and Input Space 2 jointly undergo chemical reactions in the Synthesis Space and they are integrated into new information. This process completes the projection of "xiang" from the Input Space 1 (the source domain) to the Input Space 2 (the target domain), from sensory perception to psychological perception, and from the concrete domain to the abstract domain. Along with the conceptual

2 Austin (1955) proposes "Speech Act Theory", which divides the sentence into statement sentences and performance sentences. 
integration, the semantics of "xiang" has been generalized and virtualized, and then the constructional development and evolution of "Ta bu xiang ma" realized. The process of conceptual integration of "Ta bu xiang ma" is shown in Figure 1.

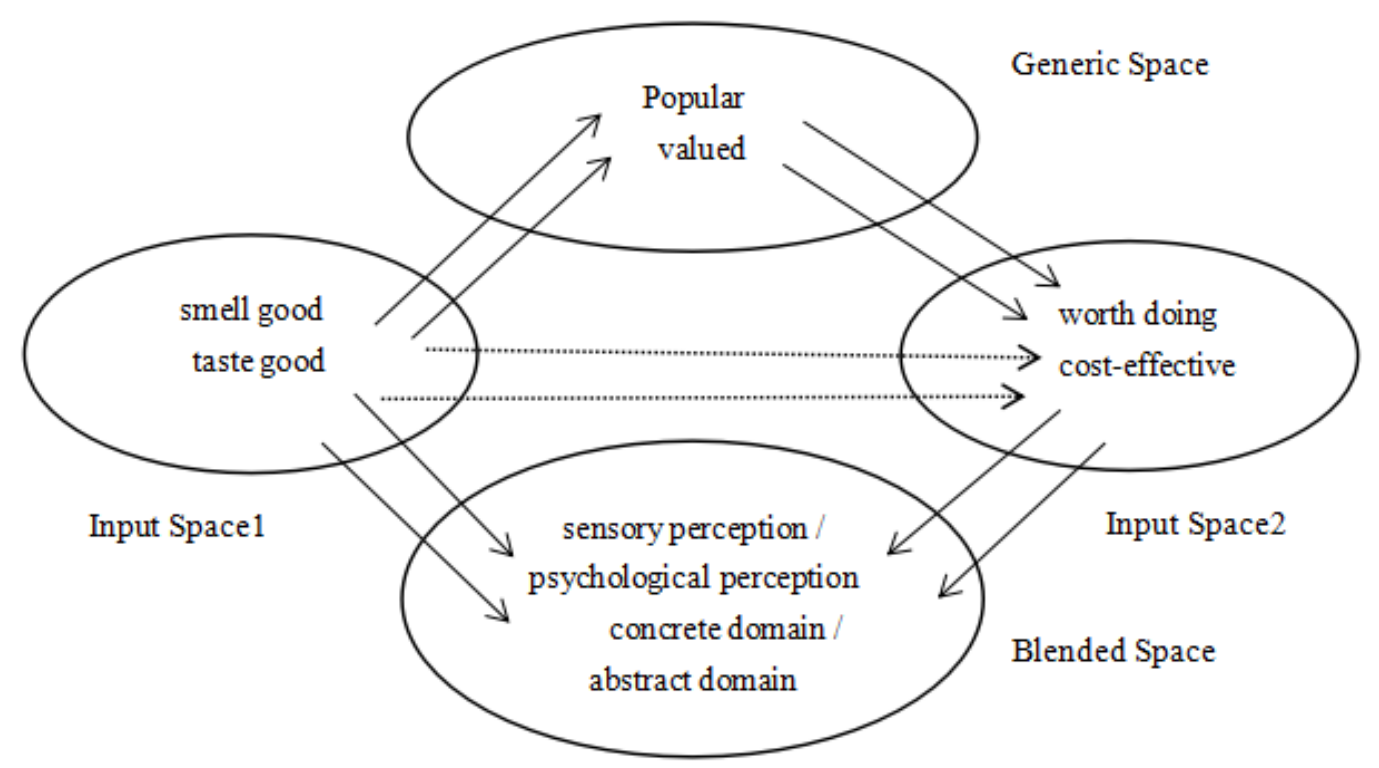

Figure. 1. Conceptual integration of "Ta bu xiang $\mathrm{ma}_{2}$ "

\section{B. Motivation}

"Ta bu xiang $\mathrm{ma}_{2}$ " is an internet buzzword. Its popularity and structural formation are mainly due to the popularity of Internet. Internet has accelerated the spread of language and the scope of its application. Since its inception, "ta bu xiang $\mathrm{ma}_{2}$ " has been favored by a large number of netizens and its use has been spread quickly. Its use has gradually penetrated into people's daily life. High-frequency use is one of the important factors for the construction and grammaticalization of language expression. High-frequency repeated use will wear out and blur the semantic meaning, and at the same time produce new syntax and text functions (Yao Shuangyun \& Yao Xiaopeng, 2012). When "ta bu xiang ma $_{2}$ " appears in example (4), the speech recipient generally does not associate "set up a stall" with fragrance that the senses can perceive, but will directly deduce the pragmatic meaning of "ta bu xiang ma" according to the context_-isn't it cost-effective, isn't it good? Over time, when the speech recipient frequently sees or hears the expression "ta bu xiang ma", he no longer needs pragmatic reasoning to think of the meaning of "cost-effective, worthwhile, and good". As the frequency of use increases, the meaning has gradually become routine and standardized, and "ta bu xiang $\mathrm{ma}_{2}$ " has also achieved a structural evolution.

（4）别去摆地摊致富了 游戏陪练它不香么? （太平洋电脑网 2020-06-11）

Bie qu baiditan zhifu le youxi peilian ta buxiang ma?

Not go set up a stall game sparring it not fragrant?

Don't go to the street stall to get rich, isn't it good to play games? (Pacific Computer Network 2020-06-11)

In addition, the psychology of seeking novelty and difference is also one of the motivations of the constructional evolution of "ta bu xiang ma,". People usually have curiosity and yearn for all the new and beautiful things, especially young people like to accept new things. "Ta bu xiang ma," uses the well-known negative rhetorical question as the structure and "xiang" as the component, conveying the defamiliarization ideological function, and realizing the organic combination of defamiliarization and familiarization. Compared with the traditional expressions, this expression can play a more unconventional effect, and are more popular among young people, especially those who pursue fashion.

\section{CONTEXT AND ITS RESPONSE}

Through collecting, sorting and analyzing the corpus, it is found that "ta bu xiang ma ${ }_{2}$ " has a strong spoken language style, and is generally used in interactive and evaluative contexts, often in natural spoken conversations, Weibo, and headlines.The basic unit of dialogue is turn-taking. According to the Conversation Analysis Theory, two adjacent turn-takings form an Adjacency Pair, in which the first turn is the triggering words and the latter turn is the response words. In specific usage, the triggering words and the response words are not completely separated, and sometimes they

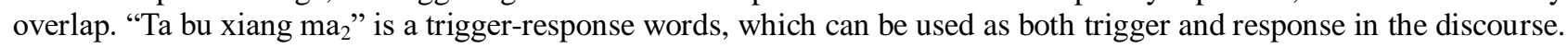
First of all, "ta bu xiang ma $_{2}$ " as a rhetorical questioning construction, it needs to respond to the rhetorical object, its triggering is non-initiating, and at the same time it will also trigger further feedback and responses from the listener. E.g: 
（5）他说: 隔壁情侣今天早上九点就起来做早饭还做了几道菜 真是精致 好幸福的感觉

我说: 周末的早上九点钟躺床睡觉不香吗 还起来做饭

他看了我一眼说: 活该你单身 (新浪微博 2020-06-20)

Ta shuo: gebi qinglv jintian zaoshang jiudian jiu qilai zuo zaofan hai zuo le jidaocai zhen shi jingzhi hao xingfu de ganjue

Wo shuo: zhoumo de zaoshang jiudianzhong tang chuang shuijiao buxiang ma hai qilai zuofan

Ta kan le wo yiyan shuo: huogai ni danshen happy feel

He say: next door couple today $9 \mathrm{am}$ already get up make breakfast make several dishes really exquisite really

I say: weekend 9am lie in the bed sleep not fragrant still get up make breakfast

He look at me say: deserve you single

He said: The couple next door got up at 9 o'clock this morning to make breakfast and cooked a few dishes. It was exquisite and so happy.

I said: Isn't it good to sleep in bed at 9 o'clock in the morning on the weekend? Get up and cook?

He glanced at me and said: You deserve to be single (Sina Weibo 2020-06-20)

"Bu xiang $\mathrm{ma}_{2}$ " in example (5) is a response to the last sentence and it also triggers the next turn. As the response, the trigger sentences of "ta bu xiang ma." are relatively simple — - the one in the conversation proposes alternatives for the other to ask. But when used as a trigger, the corresponding response has various forms.

Although Li Yuming (1990) and other researchers believe that the rhetorical question need not to answer, this is not the case in actual use. Shao Jingmin (1996) put forward that the rhetorical question is "not the kind that you do not ask others to answer, but that your answer is consistent with your own opinion". There are two types of answers, which are self-questioning answers and self-responses. The self-questioning answers include five types: "consistent answer" "reasonable answer" "inferential answer" "defensive answer" and "rebuttal answer", and self-responses answers include three types: "emphasis answer" "reasonable answer" and "effective answer".

Self-answering type of "ta bu xiang ma" is relatively infrequently used, and it is mainly used for the author's self-narrative expression and as article titles in Weibo. While the self-questioning is a category that truly reflects the interactive communication between the trigger language and the response language, this kind of question-answer model is also the main application of "ta bu xiang ma $_{2}$ " in discourse. E.g:

（6）姜子牙他不香吗? 不香不香。（爱奇艺 2020-03-08）

Jiangziya ta buxiang ma? Buxiang buxiang.

Jiang Ziya he not fragrant? Not fragrant not fragrant.

Is Jiang Ziya not good? No, it is not. (Iqiyi 2020-03-08)

（7）“我自己动手不香吗?”

“那我帮你善后。” (新浪微博 2020-08-08)

"Wo ziji dongshou buxiang ma?"

"Na wo bang ni shanhou."

"I myself do not fragrant?"

"Then I help you later."

"Isn't it good to do it myself?"

"Then I will help you aftermath." (Sina Weibo 2020-08-08)

Example (6) is an emphatic answer, using "xiang" to give a positive response to the triggering sentence. Example (7) is an inferential answer. From "then I will help you aftermath", we can deduce the speaker's attitude of supporting the other one.

\section{COMPARED WITH RELATED FoRMATS}

"Ta bu xiang $\mathrm{ma}_{2}$ " is a new and refillable construction composed of "ta" and "xiang". "Ta" is a demonstrative pronoun and its application is to make this construction more colloquial. "Ta" can also be replaced with the demonstrative pronoun "na (那)", but cannot use "zhe (这)". The reason is that although "zhe" and "na" are both demonstrative pronouns, which have substitutional and demonstrative functions, in modern Chinese their usages and frequencies are asymmetry. "Zhe" is generally used for proximal denotation, while "na' is used for the far finger (Yang Yuling, 2006). People always take themselves as the center when perceiving the external world. In comparison, things far away from the perceiving subject have low distinctiveness, which is difficult to be accurately recognized and grasped, and are often regarded as unknown things. Therefore, people usually use the pronoun "na" to relate to interrogative sentences (Zhang Zhenya, 2007). Shen Jiaxuan (1999) also pointed out that the combination of the unmarked item "zhe" and the marked item "na" with positive and negative is an unmarked combination. Therefore, "na" is closely related to the negative rhetorical question "X bu xiang ma" and can constitute an unmarked combination, forming a variant form of "ta bu xiang ma". The marked combination formed with "zhe" is not commonly used. In addition, in actual use, sometimes the demonstrative pronoun "ta" can also be omitted to form a variant of "bu xiang $\mathrm{ma}_{2}$ ", but the frequency of use is relatively low, mainly because the virtual demonstrative word "ta" also has syllables 
and Rhythm function. "Bu xiang $\mathrm{ma}_{2}$ " is a three-syllable supersegmental structure in rhetorical form, and when adding "ta" to "bu xiang ma", we can get a four-syllable form. The four-syllable form in Chinese is relatively stable and more commonly used than the three-syllable form.

The component "xiang" can also be replaced with words which have commendatory and emotional colors such as “hao (好)" and "xing (行)", but generally no derogatory words can be used. It can be explained by the "Pollyanna Hypothesis" proposed by Boucher \& Osgood (1969), that is, people always yearn for good things and try to avoid bad ones. The discourse function of "ta bu xiang ma," enables the speaker to perform the suggested speech act while uttering this sentence. The speaker generally makes reasonable and good suggestions that he thinks.

In summary, "ta bu xiang ma " can be coded into the form of "P bu X ma", and there are variants of "na bu xiang ma (那不香吗)”“"na bu hao ma (那不好吗)”“"na bu xing ma (那不行吗)”“"bu xiang ma (不香吗)”, “bu hao ma (不好 吗)"“bu xing ma (不行吗)", etc.. Each variant has the syntactic, semantic and discourse functions of “ta bu xiang ma”", but due to the different semantic accumulation of its constituent components, there are also some differences. E.g:

（8）为什么都推荐电商第三方仓储, 自建仓储他不香吗？（松松供应链 2020-05-13）

Weishenme dou tuijian dianshang disanfang cangchu, zijian cangchu ta buxiang ma?

Why all recommend e-commerce third-party warehouses? Self-built warehouses it not fragrant?

Why do people all recommend e-commerce third-party warehouses? Self-built warehouses are not popular? (Songsong Supply Chain 2020-05-13)

In the above example, "ta bu xiang ma" can be replaced with its variants and the meaning expressed is basically unchanged. However, they have subtle differences. "Ta bu xiang ma" makes suggestions in a funny way, "ta bu hao ma" emphasizes the good effect, and "Ta bu xing ma" emphasizes feasibility.

\section{CONCLUSION}

With the rise of interactive linguistics and the deepening of research, language phenomena in actual use have gradually attracted the attention of linguists. Chafe (1994) pointed out that the actual discourse that occurs naturally is the research focus of linguists. More and more Chinese researchers are studying and discussing natural spoken Chinese from the perspective of interactive linguistics. Oral communication is random, dynamic and interactive, and the language style that best reflects the interaction is the dialogue style (Fang Mei, 2007). This paper studies the formation of the new polysemous isomorphic construction "ta bu xiang ma " $_{2}$ as well as its function and application in discourse, from the perspective of interactive communication. The research found that "ta bu xiang ma" ${ }_{2}$ " is a catchword on the Internet. It has been constructed into a new and fillable construction with the rhetorical question as the structure and "ta" and "xiang" as components. Its main formation mechanism is metaphor. The development of the Internet and the widespread use of online languages, as well as people's psychology of pursuing novelty and differences are the driving factors of its construction. "Ta bu xiang $\mathrm{ma}_{2}$ " has the semantic features of subjectivity and interactive subjectivity, exclamation, anticipation and dominance. It can be used as both triggering words and response words by young people in interactive and evaluative contexts. "Ta bu xiang $\mathrm{ma}_{2}$ " has similarities and differences with its related formats. In actual use, sometimes they can be used interchangeably.

The new construction "ta bu xiang ma" is novel, but it has not yet developed into a lingua franca. Therefore, as far as teaching Chinese as a foreign language is concerned, this construction only requires students to understand, and its application is not encouraged. However, as the popularity rate increases, when it becomes a standard term, it will be included in the basic terminology that international students should master, and it is required to be able to use it while understanding it.

\section{REFERENCES}

[1] Chafe, W. (1994). Discourse, Consciousness, and Time. Chicago: Chicago University Press.

[2] Dictionary editing room, Institute of Linguistics, Chinese Academy of Social Sciences. (2016). Modern Chinese Dictionary (Seventh Edition) (Xiandai Hanyu Cidian (Di Qi Ban)). Beijing: The Commercial Press.

[3] Li Yufeng. (2010). Echoing types and negative interpretations in rhetorical questions (Fanwen de Huiying Leixing yu Fouding Yiyi). Studies of the Chinese Language, 2, 114-123.

[4] Li Yuming. (1990). The structure and understanding of rhetorical question (Fanwenju de Goucheng jiqi Lijie). Yindu Journal, 3 , 91-99.

[5] Liu Yuehua. (2001). Practical Modern Chinese Grammar (Shiyong Xiandai Hanyu Yufa). Beijing: The Commercial Press.

[6] Lv Shuxiang. (1982). Essentials of Chinese Grammar (Zhongguo Wenfa Yaolue). Beijing: The Commercial Press.

[7] Shao Jingmin. (1996). Research on Interrogative Sentences in Modern Chinese (Xiandai Hanyu Yiwenju Yanjiu). Shanghai: East China Normal University Press.

[8] Shen Jiaxuan. (2003). Compound sentences in three conceptual domains: acting, knowing, and uttering (Fuju Sanyu "Xing, Zhi, Yan”). Studies of the Chinese Language, 3,195-204.

[9] Shen Jiaxuan. (2015). Asymmetry and Markedness Theory (Bu Duichen he Biaojilun). Beijing: The Commercial Press.

[10] Wang Yin. (2007). Cognitive Linguistics (Renzhi Yuyanxue). Shanghai: Shanghai Foreign Language Education Press.

[11] Yang Yuling. (2006). A study on the asymmetry of individual zhe and individual na (Dange "zhe" he "na" Pianzhang Bu Duichen Yanjiu). Chinese Teaching in the World, 4, 33-41+147.

[12] Yao Shuangyun \& Yao Xiaopeng. (2012). On the emerging of a new function of jiushi as discourse marker in authentic speech 
(Ziran Kouyu zhong “Jiushi” Huayu Biaoji Gongneng de Fuxian). Chinese Teaching in the World, 1, 77-84.

[13] Zhang Zhenya. (2007). A functional explanation of the distributional dissymmetry between $\mathrm{Zhe}$ 这 and $\mathrm{Na}$ 那 (“Zhe/Na” $\mathrm{Bu}$ Duichen de Gongneng Jieshi). Journal of East Linguistics, 11, 13-124.

[14] Zhu Jun. (2013). "You shen me X" and "mei (you) shen me X": A case study of pragmatic negation in interactive modes(Fanwen Geshi "You shenme" de Fouding Moshi yu Fouding Dengji-Hudong Jiaoji Moshi zhong de Yuyong Fouding Gean Fenxi). Studies of the Chinese Language, 6, 505-517.

Min Zhao (b. 1980) is a PhD. student in Shanghai International Studies University. Her research interests include Linguistics and Applied linguistics. Her publications include "An Analysis of the Style and Expression of the Governance of China-Take exclaiming expression as an example" (2021), "The anti-expected expression of the interjection 'ah"” (2021), etc. 